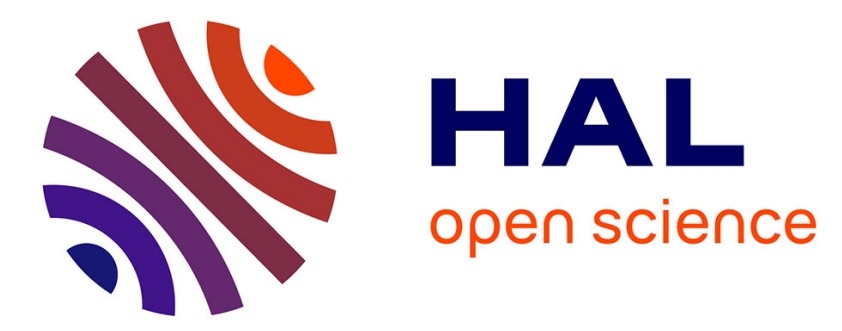

\title{
Probabilistic VOR error due to several scatterers - Application to wind farms
}

Rémi Douvenot, Ludovic Claudepierre, Alexandre Chabory, Christophe Morlaas

\section{- To cite this version:}

Rémi Douvenot, Ludovic Claudepierre, Alexandre Chabory, Christophe Morlaas. Probabilistic VOR error due to several scatterers - Application to wind farms. EUCAP 2017 11th European Conference on Antennas and Propagation, Mar 2017, Paris, France. 10.23919/EuCAP.2017.7928653 . hal-01527006

\section{HAL Id: hal-01527006 \\ https://hal-enac.archives-ouvertes.fr/hal-01527006}

Submitted on 28 May 2017

HAL is a multi-disciplinary open access archive for the deposit and dissemination of scientific research documents, whether they are published or not. The documents may come from teaching and research institutions in France or abroad, or from public or private research centers.
L'archive ouverte pluridisciplinaire HAL, est destinée au dépôt et à la diffusion de documents scientifiques de niveau recherche, publiés ou non, émanant des établissements d'enseignement et de recherche français ou étrangers, des laboratoires publics ou privés. 


\title{
Probabilistic VOR Error Due to Several Scatterers - Application to Wind Farms
}

\author{
Rémi Douvenot ${ }^{1,2}$, Ludovic Claudepierre ${ }^{1,2}$, Alexandre Chabory ${ }^{1,2}$, Christophe Morlaas ${ }^{1,2}$ \\ ${ }^{1}$ ENAC, TELECOM, F-31055 Toulouse, France \\ ${ }^{2}$ Univ de Toulouse, F-31400 Toulouse, France \\ remi.douvenot@recherche.enac.fr
}

\begin{abstract}
This paper introduces a method to calculate the VOR error due to multipaths from several known scatterers within known quantiles. In such a configuration, the amplitudes of the multipaths can be numerically or analytically calculated, whereas their phases are modelled as uniformly distributed. A probabilistic formulation of the VOR error that overestimates its variance is introduced to obtain the quantiles. The method is useful to obtain probabilities of occurrence of large VOR errors for multiple configurations and in a short computation time. Examples with wind farms are presented.
\end{abstract}

Index Terms-quantiles, VOR error, multipaths, wind turbines.

\section{INTRODUCTION}

The erection of buildings nearby VOR (VHF Omnidirectional Range) stations and radar systems are a serious concern for the air transport community. For instance, the growing development of wind farms nearby VOR stations induces bearing error for aircraft. This error has to be anticipated to allow or forbid the construction of wind farms near the stations.

Several modelling techniques have been proposed during the last decade to calculate the VOR error from the multipath. Some of them include a simple model of the CVOR (Conventional VOR) receiver [1] to quantify the VOR error [2], [3], [4]. They suppose the knowledge of the amplitude and phase of each multipath. The drawback of these approaches is that the obtained VOR error is deterministic. Therefore, the unavoidable uncertainties on the phases make the VOR error hard to interpret [4].

Usually, for a building project to be accepted by air transport regulation, one must ensure that during any in-flight test, the measured VOR error is smaller than a given threshold (typically $3^{\circ}$ ). This error can be exceeded on few positions (typically $5 \%$ of the trajectory). Quantiles perfectly fit for this type of information.

In this paper, we introduce random variables in the VOR error due to multipaths with a deterministic modelling of the multipath amplitudes and uniformly distributed phases. We obtain quantiles on the VOR error that are easy to interpret. The difficulty to define the quantiles is that the multipaths have different amplitudes, which prevents from using the central limit theorem (CLT). An approximation of the VOR error is proposed in this paper to overcome this difficulty.

First, the formulation of the VOR error is introduced. The CVOR is studied here. The adaptation to DVOR (Doppler
VOR) is straightforward. Then, the quantiles are defined for multipaths having all the same amplitude. An approximate expression of the VOR error when the amplitudes of the multipaths are different is introduced. It is used to obtain quantiles of the VOR error. Finally, simulation results are presented to show the relevancy of the proposed probabilistic approach.

\section{FORMULATION OF THE QUANTILES FOR THE VOR ERROR}

\section{A. Deterministic expression of the VOR error}

According to Odunaiya and Quinet [1], with a simple CVOR receiver model, the VOR error $\varepsilon$ due to $N$ multipaths can be approximated by

$$
\varepsilon=\tan ^{-1}\left(\frac{\sum_{n=1}^{N} a_{n} \cos \left(\theta_{n}-\theta_{0}\right) \sin \left(\varphi_{n}-\varphi_{0}\right)}{1+\sum_{n=1}^{N} a_{n} \cos \left(\theta_{n}-\theta_{0}\right) \cos \left(\varphi_{n}-\varphi_{0}\right)}\right),
$$

where $\theta_{0}$ and $\varphi_{0}$ are the phase and the azimuth of the direct signal (from the VOR station to the aircraft). $\theta_{n}$ and $\varphi_{n}$ are the phase and the azimuth of the $n^{\text {th }}$ multipath. $a_{n}$ represents the amplitude of the $n^{\text {th }}$ multipath normalised by the amplitude of the direct signal.

The linear approximation, valid for $a_{n} \ll 1$, is used in this work:

$$
\varepsilon \approx \sum_{n=1}^{N} a_{n} \cos \left(\theta_{n}-\theta_{0}\right) \sin \left(\varphi_{n}-\varphi_{0}\right)
$$

In the air transport context, we look for the maximal value of the VOR error. We choose the worst case concerning the orientation of the scatterers. It corresponds to $\sin \left(\varphi_{n}-\varphi_{0}\right)=$ $1, \forall n$, such that

$$
\varepsilon \approx \sum_{n=1}^{N} a_{n} \cos \left(\theta_{n}-\theta_{0}\right) .
$$

If one wants a finer information, the sin could be gathered with the multipath amplitude. In this case, the method presented in this paper could be used by replacing $a_{n}$ by $a_{n}^{\prime}=a_{n} \sin \left(\varphi_{n}-\right.$ $\left.\varphi_{0}\right)$ in (3).

From now on, we suppose that the normalised multipath amplitudes $a_{n}$ (or $a_{n}^{\prime}$ ) are known. In the numerical simulations presented in this paper, they are calculated with a deterministic method based on a hybridisation between physical optics and 
parabolic equation solved by split-step Fourier [4]. However, any method giving the $a_{n}$ (or an overestimation of the $a_{n}$ ) can be used.

\section{B. Probabilistic VOR error for one multipath}

We assume first that there is one multipath. We suppose that $\theta_{1}$ is a random variable uniformly distributed in $[0, \pi]$, the cos function being even. $X$ is the random variable such that $X=\cos \theta$. Then, $X$ follows the arccos distribution with the cumulative distribution function

$$
F_{X}(x)=\frac{\arccos (x)}{\pi}, \forall x \in[-1 ; 1],
$$

and the probability density function

$$
\left.f_{X}(x)=\frac{1}{\pi \sqrt{1-x^{2}}}, \forall x \in\right]-1 ; 1[.
$$

The mean and variance of $X$ are 0 and $1 / 2$, respectively. Therefore, the probabilistic VOR error with one multipath follows the distribution

$$
\left.g_{1}(x)=\frac{a_{1}}{\pi \sqrt{1-x^{2}}}, \forall x \in\right]-1 ; 1[,
$$

of null mean and variance $a_{1}^{2} / 2$.

\section{Probabilistic VOR error for $N$ multipaths of the same amplitude}

In this section, we suppose that there are $N$ multipaths with the same amplitude: $a_{n}=a_{1}, \forall n$. Then, the VOR error becomes

$$
\varepsilon=a_{1} \sum_{n=1}^{N} \cos \left(\theta_{n}-\theta_{0}\right) .
$$

Therefore, the error follows the probabilistic distribution

$$
S_{1}(x)=\sum_{n=1}^{N} a_{1} X_{n}=a_{1} \sum_{n=1}^{N} X_{n},
$$

with $X_{n}=X, \forall n$. All the multipaths have the amplitude $a_{1}$. The variance of $S_{1}$ is then $N a_{1}^{2} / 2$.

In this case, the term $\sum_{n=1}^{N} X_{n}$ is the sum of independent and identically distributed (iid) random variables. For low numbers of scatterers (up to 10), the quantiles can be tabulated. For large numbers of scatterers, the CLT is used to assimilate the sum to a Gaussian distribution. The convergence to a Gaussian distribution can be shown with quantile-quantile plots. From this step, the quantiles on the error are easily obtained.

\section{Probabilistic VOR error for $N$ multipaths with different amplitudes}

If the multipaths have different amplitudes, the sum follows the probabilistic distribution

$$
S(x)=\sum_{n=1}^{N} a_{n} X_{n} .
$$

In this formula, the multipaths follow different probabilistic distributions that cannot be tabulated ( $a_{n}$ is unknown a priori).
The CLT cannot be used since the iid hypothesis is not fulfilled. A first solution is to consider

$$
S_{\max }(x)=\sum_{n=1}^{N} a_{\max } X_{n},
$$

with $a_{\max }=\max a_{n}$. The random variables $X_{n}$ are supposed iid. Then, the previous method can be employed. The variance of $s_{\max }$ is $N a_{\max }^{2} / 2$. Therefore, this simplification drastically overestimates the real quantiles if the multipaths have different amplitudes.

However, a method to obtain finer quantiles is proposed now. Let us define an approximation of the VOR error $\tilde{\varepsilon}$ by

$$
\begin{aligned}
\tilde{\varepsilon} & =\left(\sum_{n=1}^{N} a_{n}\right)\left(\sum_{n=1}^{N} \frac{1}{N} \cos \left(\theta_{n}-\theta_{0}\right)\right) \\
& =\varepsilon_{\max }\left(\sum_{n=1}^{N} \frac{1}{N} \cos \left(\theta_{n}-\theta_{0}\right)\right) .
\end{aligned}
$$

$\varepsilon_{\max }$ is defined as the sum of the amplitudes of all the multipaths. Note here that $\tilde{\varepsilon}$ is not an overestimation of $\varepsilon$.

From $\tilde{\varepsilon}$, we define the random variable $\tilde{S}$ as

$$
\tilde{S}=\varepsilon_{\max }\left(\sum_{n=1}^{N} \frac{1}{N} X_{n}\right) .
$$

We have

$$
\begin{aligned}
\operatorname{Var}(\tilde{S}) & =\varepsilon_{\max }^{2} \operatorname{Var}\left(\sum_{n=1}^{N} \frac{1}{N} X_{n}\right)=\varepsilon_{\max }^{2} \frac{1}{N^{2}} \frac{N}{2} \\
& =\frac{1}{2 N} \varepsilon_{\max }^{2} .
\end{aligned}
$$

Therefore,

$$
\operatorname{Var}(\tilde{S}) \geq \frac{1}{2} \sum_{n=1}^{N} a_{n}^{2}=\operatorname{Var}(S)
$$

The probabilistic distribution $\tilde{S}$ defined in (12) is a product of a deterministic and a probabilistic terms. Quantiles are obtained from the probabilistic term (tabulated for $N \leq 10$ and given by the CLT for $N>10$ ). A weighting is then applied with the deterministic term. As $\operatorname{Var}(\tilde{S}) \geq \operatorname{Var}(S)$, the quantiles are overestimated with respect to the real sum (9), which is relevant with the air transport context.

Of course, the quantiles obtained with $S_{\max }$ are greater than the ones obtained with $\tilde{S}$ (equal if all the multipaths have the same amplitude).

\section{E. Quantiles for multipath power fading}

Note that a similar strategy can be adopted to calculate the attenuation power within a given quantile due to $N$ independent multipaths of known amplitudes. It is even easier since the quantiles are directly obtained from the sum of the uniform distributions of the phases. 


\section{Simulation RESUlts}

\section{A. Methodology}

All the following numerical simulations are performed using PEPO method (hybridisation parabolic wave equation / physical optics) [4]. The application cases involve wind farms near a CVOR station.

The adopted method is the following: several wind turbines are arbitrarily placed near a CVOR station. For each wind turbine, the maximum amplitude of the multipath due to this windturbine along the trajectory is obtained with PEPO. Then, the VOR error due to all the wind turbines is modelled with PEPO along the trajectory where the VOR error is the largest. Then, we look at the statistical behaviour of this error, and compare it with the quantiles computed with expression (11).

\section{B. Test case 1: circular wind farm around the VOR station}

The first scenario is illustrated in Figure 1. The VOR station is at coordinates $(0,0) .1$ to 50 wind turbines are circularly placed around the station at $10 \mathrm{~km}$ (each cross represents a wind turbine in the figure). The VOR error is calculated using the PEPO method along the trajectory in blue, from coordinates $(0,0) \mathrm{km}$ to coordinates $(65,0) \mathrm{km}$. The altitude of the aircraft is $3600 \mathrm{ft}(1097 \mathrm{~m})$.

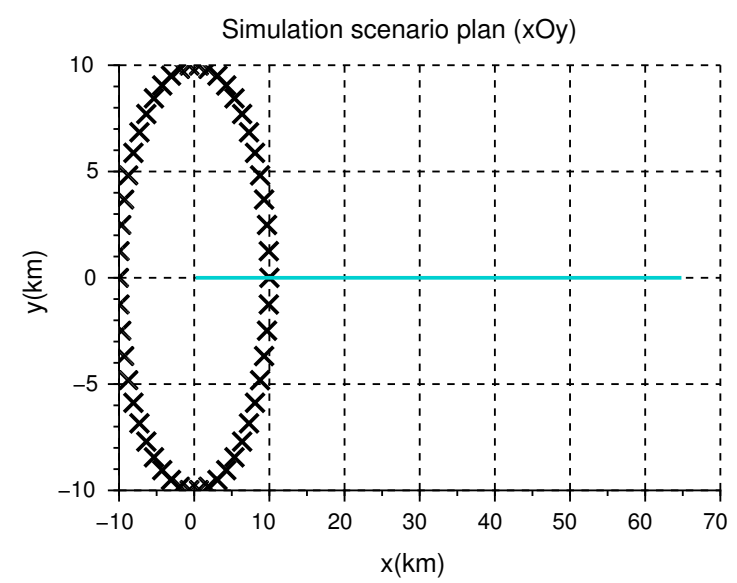

Fig. 1. Geometry of the first test case. The VOR station is at the origin. The crosses represent wind turbines. The blue line is the trajectory chosen for the simulation.

We focus on the distances $\rho 10$ to $35 \mathrm{~km}$, where the envelop of the error is the strongest. From this simulation, repeated with 1 to 50 wind turbines, the probabilistic and deterministic methods can be compared. The example of the VOR error simulated along the trajectory with 50 wind turbines is plotted in Figure 2.

In Figure 3, several VOR error levels are plotted with respect to the number of wind turbines. $\varepsilon_{\max }$, corresponding to the maximum value of the VOR error is plotted in black. The theoretical quantiles at $90 \%$ and $95 \%$ are plotted in green and red, respectively. For example, when there are 20 wind turbines, the error is less than $1^{\circ}$ at least $90 \%$ of the cases. It is compared to the values obtained from the deterministic

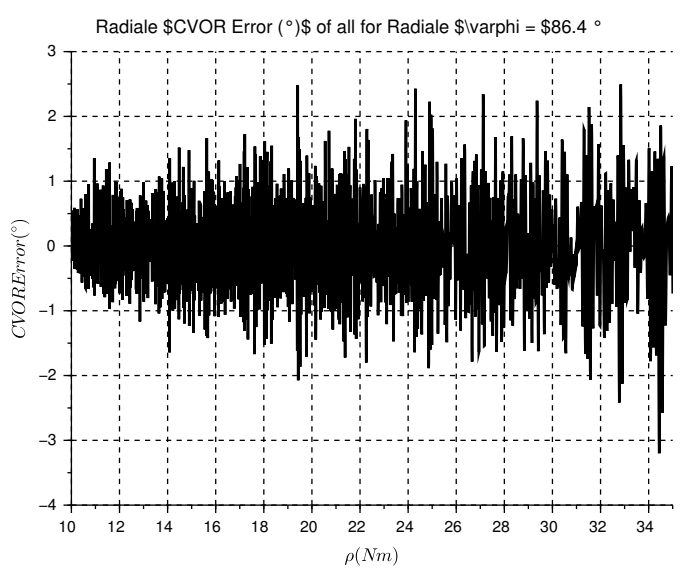

Fig. 2. VOR error simulated with PEPO in the circular configuration with 50 wind turbines with respect to the distance $\rho$ from the VOR station.

case, in blue and purple. To obtain the deterministic $90 \%$ plot, we seek from the simulated VOR error along the trajectory (Figure 2 for example) what is the maximum error when the $10 \%$ largest are removed. This is repeated for 1 to 50 wind turbines. The deterministic $95 \%$ plot is obtained the same way.

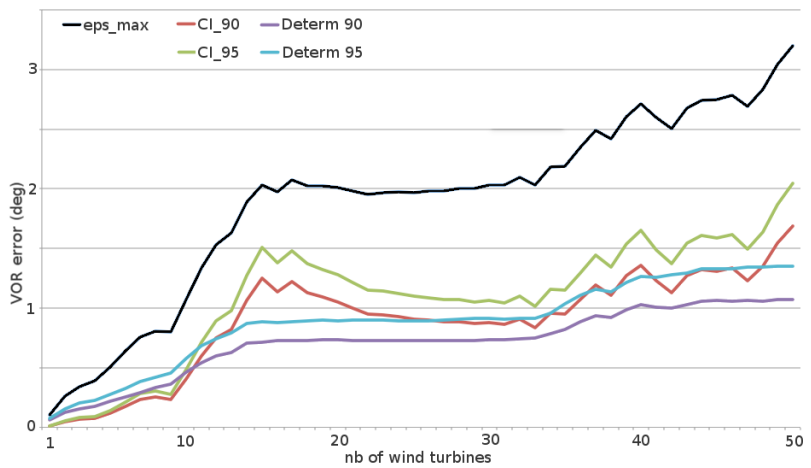

Fig. 3. Quantiles at $90 \%$ and $95 \%$, and maximum error. Probabilistic expression and estimated with PEPO.

The probabilistic method is validated if the quantiles always overestimate the deterministic result. For large numbers of windturbines, the phases are random enough, and the method is successful. For few wind turbines, we suspect that the very regular geometry goes against the iid hypothesis on the phases.

\section{Test case 2: wind farm away from the VOR station}

For this second test case, the wind farm is away from the station, between 5 and $6 \mathrm{~km}$, as seen in Figure 4. It corresponds to a more realistic case. The VOR error is simulated along the trajectory represented in blue. The corresponding simulated VOR error is represented in Figure 5.

From this simulation, the same results on the quantiles are plotted in Figure 6 (see section III-B for the details). Only the 


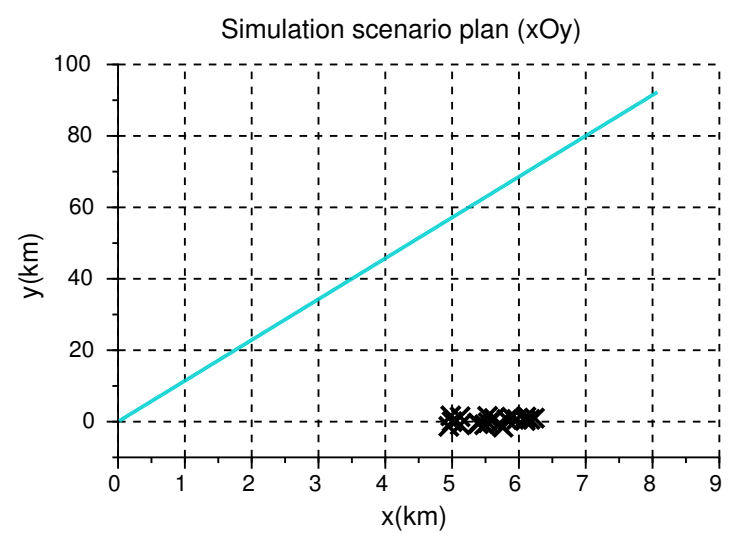

Fig. 4. Geometry of the second test case. The VOR station is at the origin The crosses represent wind turbines.

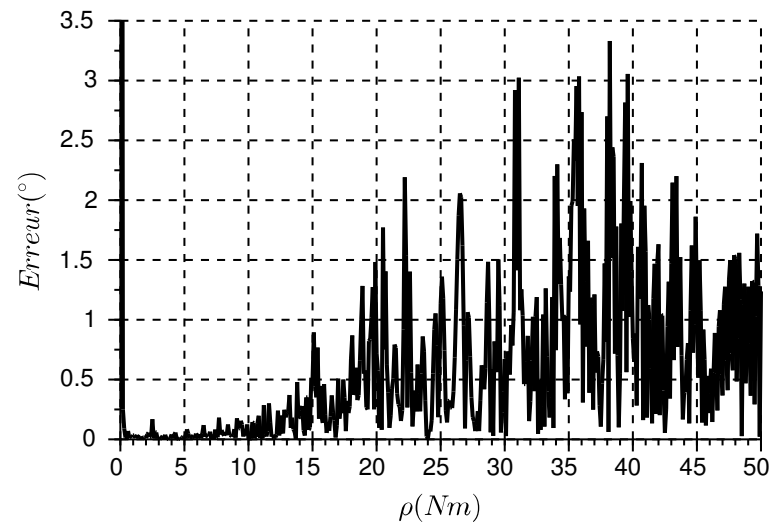

Fig. 5. VOR error simulated with PEPO in the configuration of the distant wind farm with 50 wind turbines with respect to the distance $\rho$ from the VOR station.

35 first NM (Nautic Miles) are kept, where eq. (3) is valid. After that distance, the expression cannot be linearised. Indeed, the direct signal is weak because the antenna pattern towards the trajectory after $35 \mathrm{NM}$ is low. Note that the areas where the direct signal is weak are of no practical interest.

Compared to the previous result, the interval confidence at $99 \%$ and the max error obtained with PEPO have been added. Note that the quantiles are very close to the results obtained with the deterministic method. Moreover, taking the maximum value $\varepsilon_{\max }$ drastically overestimates the VOR error along the trajectory. Note that the results for the $99 \%$ interval confidence are not overestimated. This is because there is not enough points corresponding to the $1 \%$ strongest error to have statistically significant results.

Consequently, the method proposed here is promising to obtain an good idea of the VOR error due to numerous scatterers with low modelling needs. Indeed, any method giving the amplitude of the multipath can be used as the input of the probabilistic method. Moreover, in the case of the wind farms, the quantiles answer the operational need.

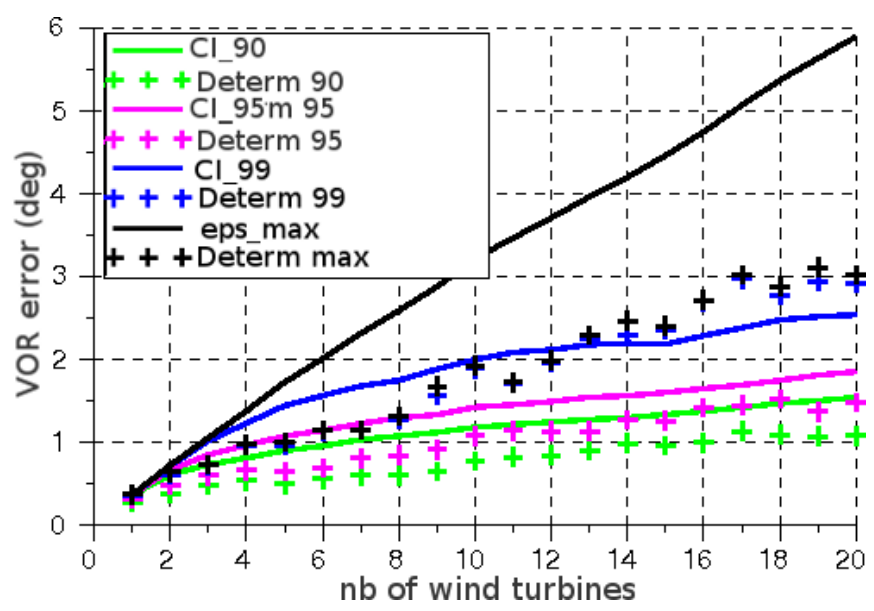

Fig. 6. Quantiles at $90 \%, 95 \%$, and $99 \%$ and maximum error. Probabilistic expression (-) and estimated with PEPO (+).

\section{CONCLUSiON}

This paper introduces a strategy to obtain quantiles of the VOR error due to multiple scatterers. It is based on an approximation of the VOR error that overestimates the variance of the error, such that the quantiles obtained from the approximated error include the real ones.

Numerical simulations show the relevancy of the proposed approach.

This strategy can be easily adapted to obtain quantiles on power fading due to multipaths in the radio-communication context.

\section{ACKNOWLEDGMENT}

The authors would like to thank the French Air Navigation Service Provider (DSNA/DTI) and the French civil aviation university (ENAC) for the founding.

\section{REFERENCES}

[1] S. Odunaiya and D. Quinet, "Calculations and analysis of signal processing by various navigation receivers architectures," in The 23rd Digital Avionics Systems Conference, DASC 04., vol. 1, Oct 2004, pp. 1.D.1-1113.

[2] C. Morlaas, M. Fares, and B. Souny, "Wind turbine effects on VOR system performance," IEEE Transactions on Aerospace and Electronic Systems, vol. 44, no. 4, pp. 1464-1476, 2008.

[3] F. Pérez Fontán, D. Marote, A. Mayo, B. Sanmartin, A. Castro, and J. J. Navarro, "Assessing multipath induced errors in VOR systems using raytracing techniques in combination with detailed terrain databases," in 14th International Flight Inspection Symposium (IFIS), June 2006, pp. 91-96.

[4] L. Claudepierre, R. Douvenot, A. Chabory, and C. Morlaas, "A deterministic VOR error modelling method - Application to wind turbines," IEEE Transactions on Aerospace and Electronic Systems, 2017, to be published. 